\title{
Strength of Lime Stabilized Pavement Materials: Possible Theoretical Explanation of Empirical Dependencies
}

\author{
Edgar Daniel Rodriguez Velasquez \\ The University of Texas at El Paso, edrodriguezvelasquez@miners.utep.edu \\ Vladik Kreinovich \\ The University of Texas at El Paso, vladik@utep.edu
}

Follow this and additional works at: https://scholarworks.utep.edu/cs_techrep

Part of the Applied Mathematics Commons

Comments:

Technical Report: UTEP-CS-20-08

\section{Recommended Citation}

Rodriguez Velasquez, Edgar Daniel and Kreinovich, Vladik, "Strength of Lime Stabilized Pavement Materials: Possible Theoretical Explanation of Empirical Dependencies" (2020). Departmental Technical Reports (CS). 1402.

https://scholarworks.utep.edu/cs_techrep/1402

This Article is brought to you for free and open access by the Computer Science at ScholarWorks@UTEP. It has been accepted for inclusion in Departmental Technical Reports (CS) by an authorized administrator of ScholarWorks@UTEP. For more information, please contact lweber@utep.edu. 


\title{
Strength of Lime Stabilized Pavement Materials: Possible Theoretical Explanation of Empirical Dependencies
}

\author{
Edgar Daniel Rodriguez Velasquez ${ }^{1,2}$ and Vladik Kreinovich ${ }^{3}$ \\ ${ }^{1}$ Department of Civil Engineering \\ Universidad de Piura in Peru (UDEP) \\ Av. Ramón Mugica 131, Piura, Peru, edgar.rodriguez@udep.pe \\ ${ }^{2}$ Department of Civil Engineering \\ ${ }^{3}$ Department of Computer Science \\ University of Texas at El Paso, 500 W. University \\ El Paso, TX 79968, USA \\ edrodriguezvelasquez@miners.utep.edu,vladik@utep.edu
}

\begin{abstract}
When building a road, it is often necessary to strengthen the underlying soil layer. This strengthening is usually done by adding lime. There are empirical formulas that describe how the resulting strength depends on the amount of added lime. In this paper, we provide a theoretical explanation for these empirical formulas.
\end{abstract}

\section{Formulation of the Problem}

Need for lime stabilized pavement layers. To have a stable road, it is often necessary to enhance the mechanical properties of the underlying soil layer. The most cost-efficient way of this enhancement is to mix soil with lime (sometimes coal fly ash is also added). Water is then added to this mix, and after a few days, the upper level of the soil becomes strengthened. The needed amount of lime depends on the soil.

How to determine the optimal amount of lime. To determine the proper amount of lime, soil specimens are brought into the lab, mixed with different amounts of lime, and tested. All chemical processes become faster when the temperature increases. So, to speed up the testing process, instead of simply waiting for several weeks as in the field, practitioners heat the sample to a higher temperature, thus speeding up the strengthening process; this highertemperature speed-up is known as curing. 
Based on the testing results, we need to predict the strength of the soil in the field for different possible lime amounts - and thus, select the lime amount that guarantees the desired strength.

Need for formulas describing the dependence of strength on curing temperature and other parameters. To be able to make this prediction, we need to know how the strength depends on the lime content $L$ (which is usually measured in percentage of lime in the dry weight of the mix). To be more precise, we need a formula with one or more parameters depending on the soil. We can then:

- determine the parameters based on the testing results, and then

- use the corresponding formula to predict the soil strength.

It turns out that the resulting empirical formulas differ depending on the porosity $\eta$ of the mix, i.e., the percentage of voids in the overall volume of the soil: for different values of $\eta$, we have, in general, different dependencies on lime content $L$.

Known empirical formulas. The mix is isotropic, so its mechanical strength can be characterized by two parameters:

- unconfined compressive strength $q_{u}$ that describes the smallest value of pressure (force over area) applied at the top of a cylindrical sample at which this sample fails;

- the tensile strength $q_{t}$ is when the force is applied orthogonally to the cylinder's axis.

For both types of strength $q$, the empirical formulas describing the dependence of strength on $\eta$ and $L$ are

$$
q=c_{1} \cdot \eta^{e_{\eta}} \cdot L^{e_{L}},
$$

for some parameters $c_{1}, e_{\eta}$, and $e_{L}$; see, e.g., $[2,3,4,5,6]$.

The corresponding constant $c_{1}$ depends on the dry density $\rho$. The dependence on $\rho$ takes the form

$$
c_{1}=c_{2} \cdot \rho^{e_{\rho}}
$$

for some constants $c_{1}$ and $e_{\rho}$; see, e.g., [4]. Substituting the formula (2) into the formula (1), we get

$$
q=c_{2} \cdot \rho^{e_{\rho}} \cdot \eta^{e_{\eta}} \cdot L^{e_{L}} .
$$

The above three formulas are supported by a large amount of evidence.

Comment. The strength also depends on the curing temperature $T$ and on the duration $d$ of curing. However, the dependence of the strength on these two quantities is less well-studied; see, e.g., $[2,6]$. While there are some useful empirical formulas, these formulas are not yet in accordance with the physical meaning. For example: 
- When the curing time $d$ is set to 0 - i.e., when there is no curing at all we should expect that these formula produce the same non-cured result, irrespective of the curing temperature.

- However, for $d=0$, different existing formula lead to values differing by the order of magnitude.

Need for a theoretical justification. In real-life applications, we can have many different combinations of the values of $L, \eta$, and $\rho$, but it is not realistically possible to test them for all possible combinations of these quantities. It is therefore desirable to come up with a theoretical justification for these empirical formulas. This will make the user more confident that the formulas can be applied to different combinations of the values of these three quantities.

\section{Our Explanation}

Main idea: scale-invariance. Our objective is to explain the dependence of the strength on the porosity, lime content, and dry density - the dependencies which are confirmed by a large amount of experiments.

The main idea behind our explanation is that while we are interested in physical quantities, when we process data, what we process are numerical values of these quantities, and these numerical values depend on the choice of the measuring unit. If we replace the original measuring unit with the one which is $\lambda$ times smaller, then the physics remains the same, but each numerical value $x$ get multiplied by $\lambda: x \rightarrow \lambda \cdot x$. For example, if we replace meters with centimeters, $2 \mathrm{~m}$ becomes $100 \cdot 2=200 \mathrm{~cm}$.

Since such "scaling" does not change the physics, it is reasonable to require that all dependencies remain the same when we change the corresponding units. Of course, if we have a dependence $y=f(x)$ - e.g., that the area $y$ of a square domain is equal to the square of its side $x\left(y=x^{2}\right)$ - then, when we change the unit for measuring $x$, we should appropriately change the unit for measuring $y$. In other words, for each $\lambda>0$, there should exist a value $\mu(\lambda)$ depending on $\lambda$ such that:

- if we have $y=f(x)$,

- then we will have $y^{\prime}=f\left(x^{\prime}\right)$ for $x^{\prime}=\lambda \cdot x$ and $y^{\prime}=\mu(\lambda) \cdot y$.

How to describe scale-invariant dependencies. Substituting the expressions $x^{\prime}=\lambda \cdot x$ and $y^{\prime}=\mu(\lambda) \cdot y$ into the formula $y^{\prime}=f\left(x^{\prime}\right)$, we conclude that

$$
f(\lambda \cdot x)=\mu(\lambda) \cdot y
$$

Here, $y=f(x)$, so we conclude that

$$
f(\lambda \cdot x)=\mu(\lambda) \cdot f(x) .
$$


From the physical viewpoint, the dependence $y=f(x)$ is usually continuous. It is known (see, e.g., [1]) that every continuous solution of the functional equation (4) has the form

$$
f(x)=c \cdot x^{e}
$$

for some parameters $c$ and $e$.

How can we apply these ideas to our formulas. At first glance, the above ideas are not applicable to the formulas (1)-(3), since these formulas deal:

- not with values measured in some physical units - were scaling would make sense,

- but rather with percentages, i.e., with values for which re-scaling does not make physical sense.

However, it is possible to apply the scaling ideas if we take into account that each of these quantities is actually a ratio of physical quantities for which re-scaling makes sense:

- the lime content $L$ is the ratio of the lime volume $V_{L}$ to the overall volume $V_{d}$ of the dry mix:

$$
L=\frac{V_{L}}{V_{d}}, \text { and }
$$

- the porosity $\eta$ is the ratio of the total volume of voids $V_{v}$ to the total volume $V$ :

$$
\eta=\frac{V_{v}}{V} .
$$

From this viewpoint, for each sample:

- the dependence of the strength $q$ on the lime content takes the form

$$
q=\text { const } \cdot V_{L}^{e_{L}}
$$

- the dependence of the strength $q$ on the porosity takes the form

$$
q=\text { const } \cdot V_{v}^{e_{\eta}} ; \text { and }
$$

- the dependence of the strength $q$ on the dry density $\rho$ takes the form

$$
q=\text { const } \cdot \rho^{e_{\rho}} .
$$

In all three cases, we now have a dependence between physical quantities for which re-scaling makes perfect sense, so it is reasonable to expect that these each of these dependencies is described by the power law. 


\section{Acknowledgments}

This work was supported in part by the US National Science Foundation grants 1623190 (A Model of Change for Preparing a New Generation for Professional Practice in Computer Science) and HRD-1242122 (Cyber-ShARE Center of Excellence).

\section{References}

[1] J. Aczel and J. Dhombres, Functional Equations in Several Variables, Cambridge University Press, Cambridge, UK, 1989.

[2] N. C. Consoli, C. G. da Rocha, and C. Silvani, "Effect of curing temperature on the strength of sand, coal fly ash, and lime blends", Journal of Materials in Civil Engineering, 2014, Vol. 26, No. 8, https://doi.org/10.1061/(ASCE)MT.1943-5533.0001011

[3] N. C. Consoli, R. B. Saldanha, J. F. Novaes, and H. C. Sheuermann Filho, "On the durability and strength of compacted coal fly ash-carbide lime blends", Soils and Rocks, 2017, Vol. 40, No. 2, pp. 155-161.

[4] S. Kumar and A. Prasad, "Parameters controlling strength of red mudlime mix", European Journal of Environmental and Civil Engineering, 2019, Vol. 23, No. 6, pp. 743-757.

[5] R. B. Saldanha and N. C. Consoli, "Accelerated mix design of lime stabilized materials", Journal of Materials in Civil Engineering, 2016, Vol. 28, No. 3, https://doi.org/10.1061/(ASCE)MT.1943-5533.0001437

[6] C. Silvani, E. Braun, G. B. Masuero, and N. C. Consoli, "Behavior of soil-fly blends under different curing temperature", Proceedings of the Third International Conference on Transportation Geotechnics ICTG'2016, Procedia Engineering, 2016, Vol. 143, pp. 220-228. 